Article

\title{
Generation of Gellan Gum-Based Adipose-Like Microtissues
}

\author{
Manuela E. L. Lago ${ }^{1,2,3}$ (1), Lucília P. da Silva ${ }^{1,2}$ (D), Catarina Henriques ${ }^{1,2}$, \\ Andreia F. Carvalho ${ }^{1,2}$, Rui L. Reis ${ }^{1,2,3}$ and Alexandra P. Marques ${ }^{1,2,3, *}$ \\ 1 3B's Research Group-Biomaterials, Biodegradables and Biomimetics, Headquarters of the European \\ Institute of Excellence on Tissue Engineering and Regenerative Medicine, University of Minho, Avepark, \\ Barco, 4805-017 Guimarães, Portugal; manuela.lago@i3bs.uminho.pt (M.E.L.L.); \\ lucilia.silva@i3bs.uminho.pt (L.P.d.S.); catarinamhenriques@ua.pt (C.H.); \\ andreiacarvalho@med.uminho.pt (A.F.C.); rgreis@i3bs.uminho.pt (R.L.R.) \\ 2 ICVS/3B's-PT Government Associate Laboratory, 4806-909 Braga/Guimarães, Portugal \\ 3 The Discoveries Centre for Regenerative and Precision Medicine, Headquarters at University of Minho, \\ 4704-553 Guimarães, Portugal \\ * Correspondence: apmarques@i3bs.uminho.pt
}

Received: 30 April 2018; Accepted: 21 June 2018; Published: 27 June 2018

\begin{abstract}
Adipose tissue is involved in many physiological processes. Therefore, the need for adipose tissue-like analogues either for soft tissue reconstruction or as in vitro testing platforms is undeniable. In this work, we explored the natural features of gellan gum (GG) to recreate injectable stable adipose-like microtissues. GG hydrogel particles with different percentages of polymer $(0.5 \%$, $0.75 \%, 1.25 \%$ ) were developed and the effect of obtained mechanical properties over the ability of hASCs to differentiate towards the adipogenic lineage was evaluated based on the expression of the early (PPAR $\gamma$ ) and late (FABP4) adipogenic markers, and on lipids formation and accumulation. Constructs were cultured in adipogenic induction medium up to 21 days or for six days in induction plus nine days in maintenance media. Overall, no significant differences were observed in terms of hASCs adipogenic differentiation within the range of Young's moduli between 2.7 and $12.9 \mathrm{kPa}$. The long-term (up to six weeks) stability of the developed constructs supported its application in soft tissue reconstruction. Moreover, their ability to function as adipose-like microtissue models for drug screening was demonstrated by confirming its sensitivity to TNF $\alpha$ and ROCK inhibitor, respectively involved in the repression and induction of the adipogenic differentiation.
\end{abstract}

Keywords: adipose tissue engineering; adipogenic differentiation; adipose-like microtissues; gellan gum; hydrogels

\section{Introduction}

Adipose tissue is no longer considered solely an energy storage tissue. It is a complex tissue involved in several biological processes including the endocrine/paracrine regulation of energy metabolism and thermoregulation, that also provides key structural protection and support to major organs $[1,2]$. Increasing adipose tissue loss associated with various pathological conditions, namely oncologic resection, trauma, and congenital abnormalities, has been supporting the need for improved strategies for soft tissue reconstruction, currently limited due to high resorption rates [3]. Moreover, endocrine and metabolic diseases such as diabetes and obesity, hallmarks of developed societies, require additional knowledge to be delivered for further improving the targeted therapeutics and enhanced success rates. Considering this, new strategies to generate adipose-like tissues that can be either used in soft tissue reconstruction or as reliable in vitro models are in an undeniable demand. 
The combination of adult stem cells from various sources within 3D polymeric structures has been one of the most approached to generate 3D adipose-like tissues. The majority of these works have been using 3T3-L1 [4-7], a murine pre-adipocyte cell line that has limited representation of human tissue physiology. In alternative, adult stem cells have been differentiated into the adipogenic lineage in a range of natural/synthetic materials [8-11]. Considerations, such as the mimicking of the dimensions of the fat lobules at the microscale [8] and of adipose tissue mechanical properties, have been addressed from the material's perspective at the initial stage of development. While these have been shown to correlate with the achieved degree of differentiation, it is also known that to be reliable and functional, the generated constructs have to possess appropriate stability. Factors such as the type of material $[10,12,13]$ and respective degradation rate, in vitro culture conditions $[11,14]$ and (pre)-vascularization $[8,11]$ are among those known to influence it however, little is known about the requirements to achieve ideal stability.

Gellan gum (GG) is a natural polymer that has been proposed for various tissue engineering and regenerative medicine applications [15-18]. Because GG is thermosensitive and reacts with monovalent or divalent cations, it forms hydrogels by temperature change and by ionic crosslinking. Thus, the properties of GG hydrogels are highly tunable by changing the polymer and/or crosslinking concentration, or even crosslinking type and conditions. Moreover, GG is not susceptive to enzymatic action which makes GG hydrogels degradation dependent on hydrolytic reactions. Cell adhesiveness, except when combined with peptide sequences $[19,20]$ or processed in particular modes $[21,22]$, is not a feature naturally depicted by GG hydrogels.

Considering that along adipogenic differentiation, cells suffer continuous alterations in integrin expression and that disruption of the extracellular matrix (ECM)-cell contact is fulcra [1], we hypothesized that natural GG hydrogels features, like reduced cell-ECM interactions mimicking and degradation rate, would potentiate adipogenic differentiation of human adipose derived stem cells (hASCs). Moreover, by tuning the physic-chemical and mechanical properties of GG hydrogels we could add to the generation of stable GG-based adipose-like microtissues. Hence, GG hydrogel particles with different percentages of polymer were developed and the effect of obtained mechanical properties over the ability of hASCs to differentiate towards the adipogenic lineage was evaluated. Degradability was also assessed to assure the stability of the developed constructs, critical for its application in soft tissue reconstruction. Moreover, their ability to function as adipose-like microtissue models for drug screening was analysed by testing its sensitivity to molecules involved in the induction/repression of the adipogenic differentiation (Scheme 1).

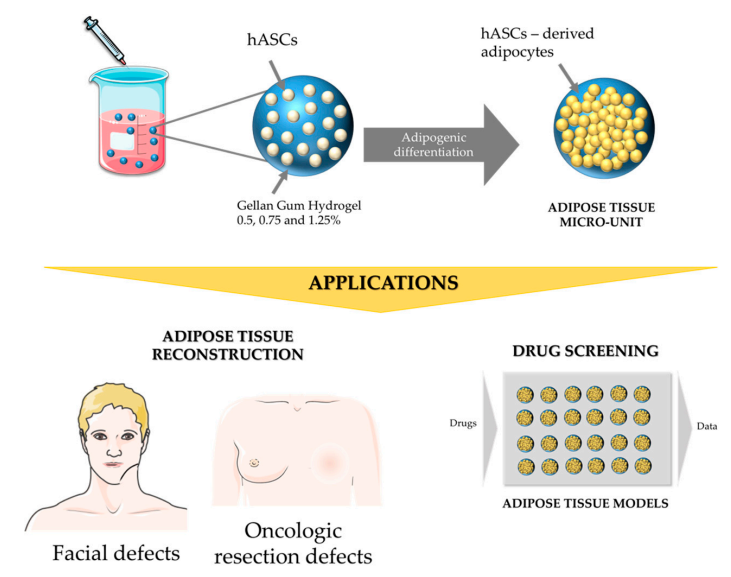

Scheme 1. Schematic representation of the development of adipose-like microtissues and potential applications. Human adipose derived stem cells (hASCs) are encapsulated in gellan gum-based hydrogel particles with different percentages of polymer, and differentiated into the adipogenic lineage. The obtained adipose-like microtissues can be used for adipose tissue reconstruction of defects from oncologic resection, trauma and congenital abnormalities, or as 3D in vitro tissue analogues for drug screening. 


\section{Materials and Methods}

\subsection{Gellan Gum Hydrogel Particles Preparation}

Gellan gum (GG) hydrogels were prepared as previously described [21] with some modifications. Gelzan (Sigma-Aldrich, Saint Louis, MI, USA) was filtered through a sterile filtration system (Techno Plastic Products AG, Trasadingen, Switzerland), frozen in liquid nitrogen and lyophilized (LyoAlfa 10/15, Telstar, Terrassa, Spain) for three days to obtain sterile, dried and purified material. This was dissolved in deionized (DI) $\mathrm{H}_{2} \mathrm{O}$ to attain a final concentration of $0.5 \%, 0.75 \%$, and $1.25 \%(w / v)$. After complete dissolution of the polymer at $90{ }^{\circ} \mathrm{C}$ under stirring, the temperature was lowered to $40{ }^{\circ} \mathrm{C}$ and Alpha Minimum Essential Medium ( $\alpha$-MEM) (Life Technologies, Paisley, UK) was added and then dispensed into a hydrophobic surface using a syringe/pipette for viscous solutions to produce spherical hydrogel particles. The size of the particles was given by the dispensed amount (1 to $50 \mu \mathrm{L})$. The injectability was assessed by coupling a $27 \mathrm{G}$ needle in a syringe $(1 \mathrm{~mL})$ by loading the particles in a GG vehicle solution. Images of the particles were acquires using a Stereo Microscope + Lamp (Schott KL 200, Stemi 1000, Carl Zeiss, Oberkochen, Germany) combined with AxioVision software (version 4.8.2.0, Carl Zeiss, Oberkochen, Germany) which allows to measure hydrogel particles diameter.

\subsection{Oscillatory Rheology}

The viscoelastic properties were determined using an oscillatory rheometer (MAL1097376, Kinexus Prot, Malvern, UK). Hydrogels discs ( $5 \mathrm{~mm} \varnothing, 4 \mathrm{~mm}$ height) were prepared as previously described for the particles but using a punctured mould and maintained in complete $\alpha$-MEM medium for two days to reach the swelling equilibrium. The discs were loaded in the rheometer between parallel plates ( $25 \mathrm{~mm}$ in diameter) adjusting the gap to reach a normal force close to $0 \mathrm{~N}$ (normal force maximum is $0.5 \mathrm{~N})$. The storage $\left(\mathrm{G}^{\prime}\right)$ and loss $\left(\mathrm{G}^{\prime \prime}\right)$ moduli were measured by performing a dynamic time sweep at a frequency of $10 \mathrm{rad} / \mathrm{s}, 0.5 \%$ strain and at $25{ }^{\circ} \mathrm{C}$ along time until a plateau was reached.

\subsection{Degradation}

The degradation of GG hydrogel particles was analysed by determining the mass loss after incubation with a $\mathrm{NaCl}(0.154 \mathrm{M})$ solution at $37^{\circ} \mathrm{C}$ and stirring $(180 \mathrm{rpm})$ for 42 days. Mass loss was determined by measuring the weight of GG hydrogels particles before (Wi) and after (Wf), immersion into the $\mathrm{NaCl}$ solution. Maximum mass loss at the end of the incubation time (six weeks) was also determined by measuring the weight of the dried GG hydrogels particles after freeze-drying at the final time-point.

Mass loss was calculated according to the following equation:

$$
\operatorname{mass}(\%)=\frac{\mathrm{Wf}-\mathrm{Wi}}{\mathrm{Wi}} \times 100
$$

where Wi represents the initial weight of the hydrogel particles and Wf represents the weight of the hydrogel particles at each time-point or the dried hydrogel particles weight at the last time-point after freeze-drying.

\subsection{Isolation and Characterization of Human Adipose Stem Cells}

Human adipose stem cells (hASCs) were isolated as previously described [23] from the subcutaneous adipose tissue of three donors that underwent liposuction procedures at Hospital da Prelada (Porto). Samples were collected with the informed consent of the patients and under a collaboration protocol with 3B's Research Group, approved by the ethical committees of both institutions. hASCs were expanded in Alpha Minimum Essential Medium ( $\alpha$-MEM) supplemented with 10\% Foetal Bovine Serum (FBS) (Life Technologies, Bleiswijk, The Netherlands) and 1\% Antibiotic/Antimycotic (ATB) (Life Technologies, Paisley, UK) in a humidified atmosphere with 
$5 \% \mathrm{CO}_{2}$ at $37^{\circ} \mathrm{C}$. The mesenchymal stem cell phenotype of hASCs was confirmed by the expression of the principal mesenchymal markers (CD90, CD105 and CD73) analysed by flow cytometry and after differentiation into the adipogenic and osteogenic lineages (Supplemental Material).

\subsection{Cell Encapsulation within Gellan Gum Hydrogels Particles}

Human adipose stem cells were resuspended in GG solution at concentrations of $3 \times 10^{3}$ cells $/ \mu \mathrm{L}$. After proper dispersion of the cells within the polymeric solution, hydrogel particles were prepared as described above. Cell-laden hydrogel particles were incubated in supplemented $\alpha$-MEM medium in a humidified atmosphere with $5 \% \mathrm{CO}_{2}$ at $37^{\circ} \mathrm{C}$.

\subsection{Adipogenic Differentiation within Gellan Gum Hydrogels Particles}

Adipogenic differentiation of hASCs within gellan gum hydrogel particles was induced as previously described [24] with some modifications. After culturing cell-laden hydrogel particles in $\alpha$-MEM medium for three days, medium was changed to an adipogenic induction medium (IM) consisting of $\alpha$-MEM supplemented with $10 \%$ FBS, $1 \%$ ATB, $34 \mu \mathrm{M}$ of D-pantothenate (Sigma-Aldrich, Sintra, Portugal) and $66 \mu \mathrm{M}$ of biotin (Sigma-Aldrich, Sintra, Portugal), $200 \mathrm{nM}$ of insulin (Sigma-Aldrich, Sintra, Portugal), $1 \mu \mathrm{M}$ of dexamethasone (Sigma-Aldrich, Sintra, Portugal), $250 \mu \mathrm{M}$ of 3-isobutyl-1-methylxanthine (IBMX) (Sigma-Aldrich, Sintra, Portugal), and $5 \mu \mathrm{M}$ of troglitazone (Sigma-Aldrich, Sintra, Portugal). Cells were cultured for further 3, 6, 15 and 21 days. An additional condition was set after days six of induction, by changing the medium to maintenance medium (MM) - IM without IBMX and troglitazone—and culture the cell-laden hydrogel particles for a further nine days.

\subsection{Incubation with TNF Alpha and ROCK Inhibitor}

To assess the influence TNF alpha (inhibitor) and Rho kinases (ROCK) inhibitor (promoter) on the adipogenic differentiation, $0.5 \%$ GG hydrogel particles with hASCs were cultured for 15 days in induction medium supplemented with $0.5 \mu \mathrm{g} / \mathrm{mL}$ of TNF $\alpha$ or $50 \mu \mathrm{M}$ of ROCK inhibitor. Control conditions in basal medium and in differentiation medium without the tested molecules were considered.

\subsection{Quantitative Real Time Polymerase Chain Reaction (RT-PCR)}

Cell-laden hydrogel particles were collected in $400 \mu \mathrm{L}$ of Tri-reagent (Sigma-Aldrich, Sintra, Portugal) and preserved at $-80{ }^{\circ} \mathrm{C}$ until extraction. For RNA extraction, samples were incubated for 5 min at RT, macerated with a tissue grinder (Nippon genetics, Duren, Germany) and subsequently centrifuged for $5 \mathrm{~min}$ at $6000 \mathrm{G}$. The pellet was discarded and $80 \mu \mathrm{L}$ of chloroform (Sigma-Aldrich, Sintra, Portugal) was added to the recovered supernatants. Following an incubation period of $15 \mathrm{~min}$ at RT, samples were centrifuged at $4{ }^{\circ} \mathrm{C}$ for $20 \mathrm{~min}$ at $13,000 \mathrm{rpm}$. After centrifugation, the aqueous phase was collected and $200 \mu \mathrm{L}$ of isopropanol (VWR, Carnaxide, Portugal) were added. Following a $10 \mathrm{~min}$ of incubation period at RT, samples were centrifuged at $4{ }^{\circ} \mathrm{C}$ for $10 \mathrm{~min}$ at $13,000 \mathrm{rpm}$. The supernatants were discarded and pellets were washed once with 100\% ethanol and twice with $70 \%$ ethanol, by centrifugation at $4{ }^{\circ} \mathrm{C}$ for $5 \mathrm{~min}$ at 13,000 rpm. Extracted RNA was kept in $10 \mu \mathrm{L}$ of RNAse/DNAse free water (Lonza, Verviers, Belgium).

RNA quantity and purity were assessed using a NanoDrop N-1000 Spectrophotometer (Thermo Fischer Scientific, Wilmington, DE, USA). Samples with a 260/280 nm ratio between 1.6 and 2.2 were used for cDNA synthesis. Synthesis was performed using a QSCript cDNA SuperMix (Quanta Biosciences, Gaithersburg, MD, USA) and a Reverse Transcription Polymerase Chain Reaction (RT-PCR) Mastercycler (Eppendorf, Hamburg, Germany). An initial amount of 200 ng of RNA in RNAse/DNAse free water was used for a total volume of $20 \mu \mathrm{L}$.

FABP4 and PPAR $\gamma$ transcripts were quantified in the cDNA samples using a Quantitative Real Time Polymerase Chain Reaction (qRT-PCR). The primers were designed using the Primer-BLAST 
tool (NCBI, Bethesda, MD, USA) and synthesized by Eurofins genomics (Ebersberg, Germany) as listed in Table 1. The Real-Time PCR reaction was done using PerfeCta SYBR Green FastMix (Quanta Biosciences, Gaithersburg, MD, USA), following manufacturer's instructions in a Reverse Transcription Polymerase Chain Reaction Mastercycler (Eppendorf, Hamburg, Germany). FABP4 and PPAR $\gamma$ were amplified for 45 cycles in a total volume of $20 \mu \mathrm{L}$. Each cycle comprised a denaturation step at $95^{\circ} \mathrm{C}$ for $10 \mathrm{~s}$, followed by an annealing step at specific temperatures (see Table 1) for $30 \mathrm{~s}$ and an extension step at $72{ }^{\circ} \mathrm{C}$ for $30 \mathrm{~s}$. Relative gene expression was analysed against GAPDH housekeeping gene.

Table 1. Primers sequences.

\begin{tabular}{|c|c|c|c|}
\hline Gene & Primer Forward $\left(5^{\prime}-3^{\prime}\right)$ & Primer Reverse $\left(5^{\prime}-3^{\prime}\right)$ & $\operatorname{Tm}\left({ }^{\circ} \mathrm{C}\right)^{1}$ \\
\hline$P P A R \gamma$ & TGGGTGAAACTCTGGGAGAT (20) & CCA (20) & 57.3 \\
\hline FABP4 & AAACTGGTGGTGGAATGCGT (20) & GCGAACTTCAGTCCAGGTCA (20) & 58.4 \\
\hline GAPDH & AGCCTCAAGATCATCAGCAA (20) & GTCATGAGTCCTTCCACGAT (20) & 56 \\
\hline
\end{tabular}

${ }^{1} \mathrm{Tm}-$ Melting temperature.

\subsection{Western Blot}

Cell-laden hydrogels were collected in radio-immunoprecipitation assay (RIPA) buffer containing protease inhibitor (1:100) and Dithiothreitol (DTT) (1:1000), all from Sigma-Aldrich (Sintra, Portugal), to reduce disulphide bonds and protect proteins from denaturation. Samples were macerated with a tissue grinder (Nippon genetics, Duren, Germany), left for $30 \mathrm{~min}$ in lysis buffer solution and centrifuged for $20 \mathrm{~min}$ at $4{ }^{\circ} \mathrm{C}$ and $12,000 \times \mathrm{g}$. Protein quantification was performed using Bradford assay kit (Thermo Fisher Scientific, Rockford, IL, USA), according to manufacturer's instructions. For Western Blot, $10 \mu \mathrm{g}$ of each samples were loaded in a 19\% SDS polyacrylamide gel (Sigma-Aldrich, Sintra, Portugal) for electrophoresis and subsequently transferred onto a nitrocellulose membrane (GE Healthcare, Buckinghamshire, UK). The blot was then incubated in Ponceau solution, and posteriorly blocked with a $5 \%$ milk powder solution for $1 \mathrm{~h}$. The blot was incubated at $4{ }^{\circ} \mathrm{C}$ overnight with a rabbit polyclonal FABP4 antibody (1:1000) and with a rabbit polyclonal beta-tubulin antibody (Abcam, Cambridge, UK) at a dilution of 1:500 (loading control). The bound antibody was detected with an anti-rabbit alkaline phosphatase secondary antibody (Sigma-Aldrich, Sintra, Portugal) diluted in a 5\% milk powder solution at 1:5000. Bands were visualized using an AP Conjugate Substrate kit (Biorad, Lisbon, Portugal), followed by scanning with an EPSON Perfection V600 Scanner (EPSON, Nagano, Japan). Band intensities were quantified using ImageJ software (Version 1.52b, NIH, Baltimore, MD, USA).

\subsection{Immunocytochemistry}

Cell-laden hydrogel particles were fixed in $10 \% v / v$ of formalin (Bio-Optic, Milano, Italy) for $1 \mathrm{~h}$, incubated with $1 \%$ Triton X-100 (Sigma-Aldrich, Sintra, Portugal) for $20 \mathrm{~min}$ at $4{ }^{\circ} \mathrm{C}$, and with $2.5 \% w / v$ of horse serum (HS, Vector Laboratories, Burlingame, CA, USA) for $1 \mathrm{~h}$, respectively for cell permeabilization and blocking of nonspecific antibody binding. Samples were then incubated with rabbit anti-human primary antibodies FABP4 (1:100), PPAR gamma (1:25) (Abcam, Cambridge, UK) diluted in $1 \%$ BSA, $0.2 \%$ Triton in PBS for $24 \mathrm{~h}$. After washing with PBS, samples were incubated overnight at $4{ }^{\circ} \mathrm{C}$ with the secondary antibody Alexa Fluor 488 donkey anti-rabbit (Life Technologies, Carlsband, CA, USA) at a concentration of 1:500 in 1\% HS in PBS. Nuclei were counter-stained with DAPI $(0.02 \mathrm{mg} / \mathrm{mL})$. Cell-laden hydrogel particles were observed using a Leica TCS SP8 confocal microscope (Leica, Mannheim, Germany) or an AxioImager Z1m fluorescence microscope (Zeiss, Gottingen, Germany). 


\subsection{Nile Red Staining and Quantification}

Cell-laden hydrogel particles, previously fixed in 10\% formalin, were placed in a diluted solution of Nile Red $\left(0.05 \mu \mathrm{g} / \mathrm{mL}, 1: 2000\right.$ dilution in PBS) and incubated for $20 \mathrm{~min}$ at $4{ }^{\circ} \mathrm{C}$. Nuclei were counter-stained with DAPI $(0.02 \mathrm{mg} / \mathrm{mL})$. Lipids were observed using a Leica TCS SP8 confocal microscope (Leica, Mannheim, Germany) or an AxioImager Z1m fluorescence microscope (Zeiss, Gottingen, Germany). ImageJ software was used to count the number of Nile Red stained cells in relation to the total number of cells (DAPI) in 9 random images for each condition.

\subsection{Statistical Analysis}

GraphPad Prism 7 software (La Jolla, CA, USA) was used to perform statistical analysis. The results were compared to a control condition corresponding to the samples before induction of the differentiation. Data was analysed using the Shapiro-Wilk normality test. Data following a normal distribution was analysed using a two-way ANOVA with Turkey post-test and a one-way ANOVA with Turkey post-test. Significance was set to ${ }^{*} p<0.05,{ }^{* * *} p<0.001$; ${ }^{* * * *} p<0.0001$ ). All quantitative data refer to $n=3$ and are presented as mean \pm standard deviation.

\section{Results}

\subsection{GG Hydrogel Particles Properties}

Considering soft-tissue reconstruction and the possibility to inject the adipose tissue-like microtissues to fill in a defect, GG spherical hydrogel particles of different sizes were produced. The size of the particles, given by the dispensed amount (from 1 to $50 \mu \mathrm{L}$ ) (Figure 1A), ranged from 700 to $5000 \mu \mathrm{m}$ in diameter, approximately (Figure 1B). To demonstrate that injection would not interfere with the integrity of the hydrogel particles, they were loaded in a GG vehicle solution and dispensed using a syringe $(1 \mathrm{~mL})$ coupled to a $27 \mathrm{G}$ needle (Figure $1 \mathrm{C})$. After injection the hydrogel particles kept the spherical shape and did not disintegrate (Figure 1C,D).

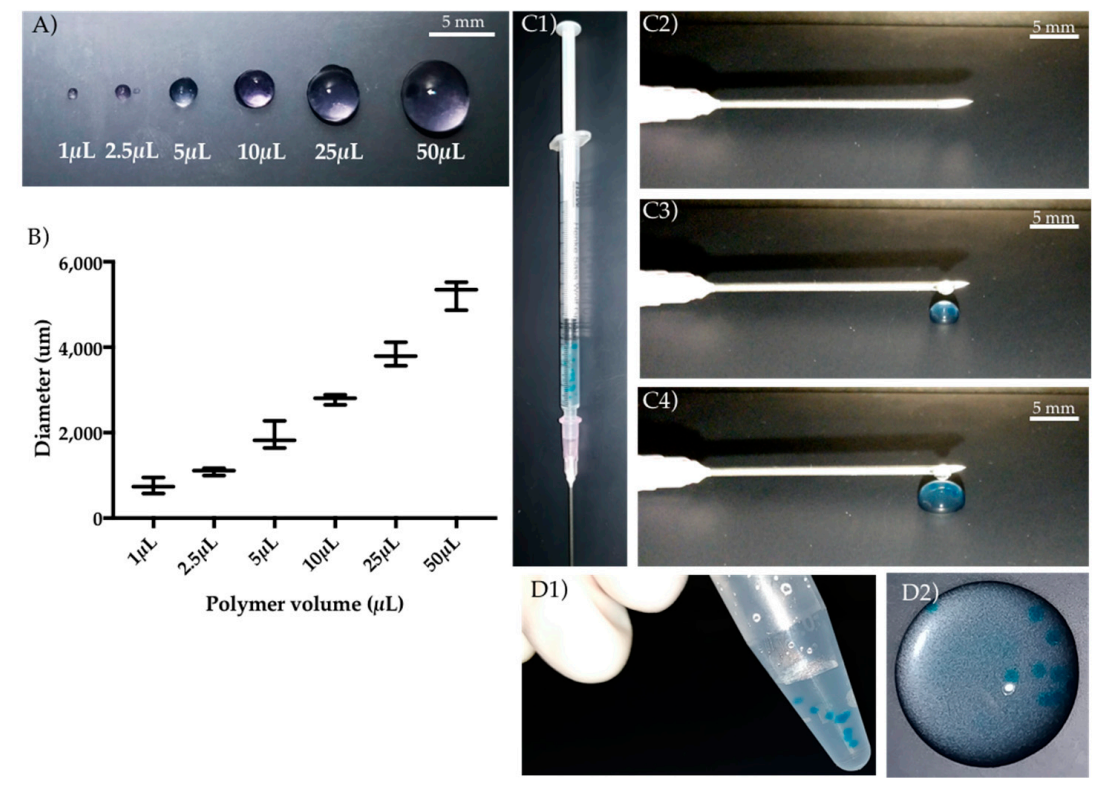

Figure 1. Production and injectability of gellan gum (GG) hydrogel particles. (A,B) Spherical particles with different sizes ranging from 700 to $5000 \mu \mathrm{m}$ of diameter, approximately are obtained by dispensing different volumes of polymer solution (from 1 to $50 \mu \mathrm{L}$ ). (C) The obtained particles (previously stained with methylene blue) are loaded in a GG solution (C1) that acts as vehicle to confirm its injectability through a needle of 27G (C2-C4). (D) The integrity of the particles before (D1) is maintained after injection (D2). 
To determine if the amount of polymer affected the mechanical properties of the hydrogel particles, the storage $\left(\mathrm{G}^{\prime \prime}\right)$ and loss $\left(\mathrm{G}^{\prime \prime}\right)$ moduli were measured (Figure 2A). The storage modulus determined by the most linear curve in a dynamic time sweep (Figure 2B) was higher for hydrogels with $1.25 \%$ of polymer content, which represents an increase of $17 \%$ and $33 \%$ in comparison to hydrogels respectively with $0.75 \%(p<0.05)$ and $0.5 \%(p<0.05)$ of polymer content.

The stability of the prepared GG hydrogel particles in saline solution was analysed based on the hydrogel particles mass loss up to 42 days (six weeks). After a fast mass loss within the first day, the profiles of the hydrogel particles kept relatively constant along the six weeks of incubation time (Figure 2C). When the dry weight of the particles was measures at the end time point, it was possible to see that the particles did not degrade and that the weight variations did not correspond to loss of polymeric content (Figure 2C,D).

A)

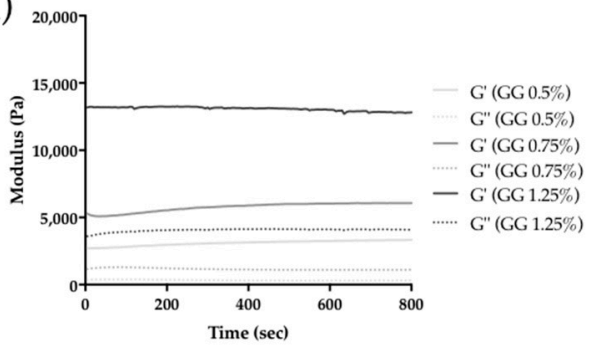

C)

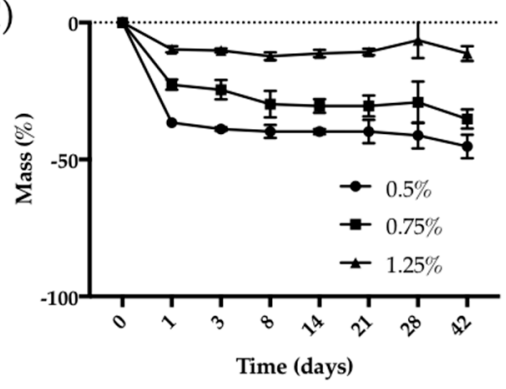

B)

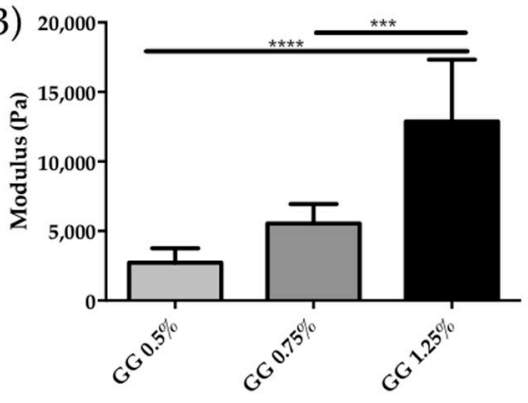

D)

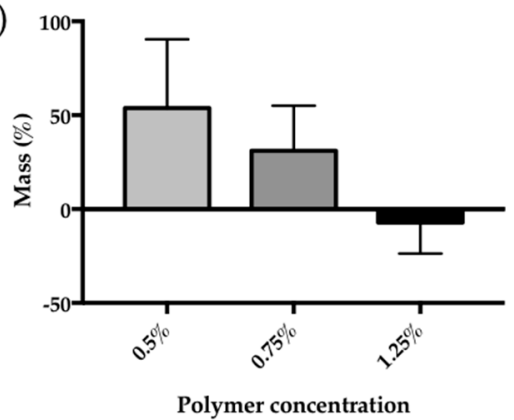

Figure 2. Physic-chemical and mechanical properties of gellan gum (GG) hydrogels. (A) Representative dynamic time sweep of storage $\left(\mathrm{G}^{\prime}\right)$ and loss $\left(\mathrm{G}^{\prime \prime}\right)$ moduli of GG hydrogels using strain of $0.5 \%$, frequency of $10 \mathrm{rad} / \mathrm{s}$ and at $25^{\circ} \mathrm{C}$. (B) Plot of storage $\left(\mathrm{G}^{\prime}\right)$ and loss $\left(\mathrm{G}^{\prime \prime}\right)$ moduli of GG hydrogels obtained from the dynamic time sweep $(n=5)$. (C) Mass loss profile of GG hydrogel particles along incubation with a saline solution for 42 days (six weeks, $n=5$ ). (D) Plot of maximum mass value after six weeks. ${ }^{* * *} p<0.001$ and ${ }^{* * * *} p<0.0001$.

\subsection{Assessment of hASCs Differentiation within GG Hydrogel Particles}

To evaluate the influence of the physic-chemical and mechanical properties of the different formulations of GG hydrogel particles on the differentiation of hASCs, the expression of the early and late adipogenic markers, respectively PPAR $\gamma$ and FABP4, and of the lipid formation and accumulation were evaluated.

The results obtained from the qPCR analysis for PPAR $\gamma$ (Figure 3A) and FABP4 (Figure 3B) demonstrated that these genes started to be expressed after six days of culture in induction medium. The expression of PPAR $\gamma$ increased for higher times of culture in induction medium up to day 15 . The switch to maintenance medium after six days of culture in induction one, did not affect the expression of PPAR $\gamma$. In opposition, PPAR $\gamma$ expression within $0.5 \%$ GG hydrogel particles significantly increased $(p<0.05)$ along the time of culture in induction medium. This tendency seems to be 
also observed within $0.75 \%$ and $1.25 \%$ GG particles up to day 15 , although without significant differences. The expression of FABP4 in the 0.5\% GG hydrogel particles showed the same trend as PPAR $\gamma$, significantly increasing $(p<0.05)$ along the time of culture in induction medium. Moreover, at day 15 the expression of PPAR $\gamma$ in the $0.5 \%$ GG hydrogel particles was significantly higher $(p<0.05)$ than in the $1.25 \%$ ones. Interestingly, independently of the concentration of the particles, the expression levels at day six in induction plus nine days in maintenance media were similar to those observed at day six only in induction medium, and significantly lower than those at day 15 within the $0.25 \%$ GG hydrogel particles.
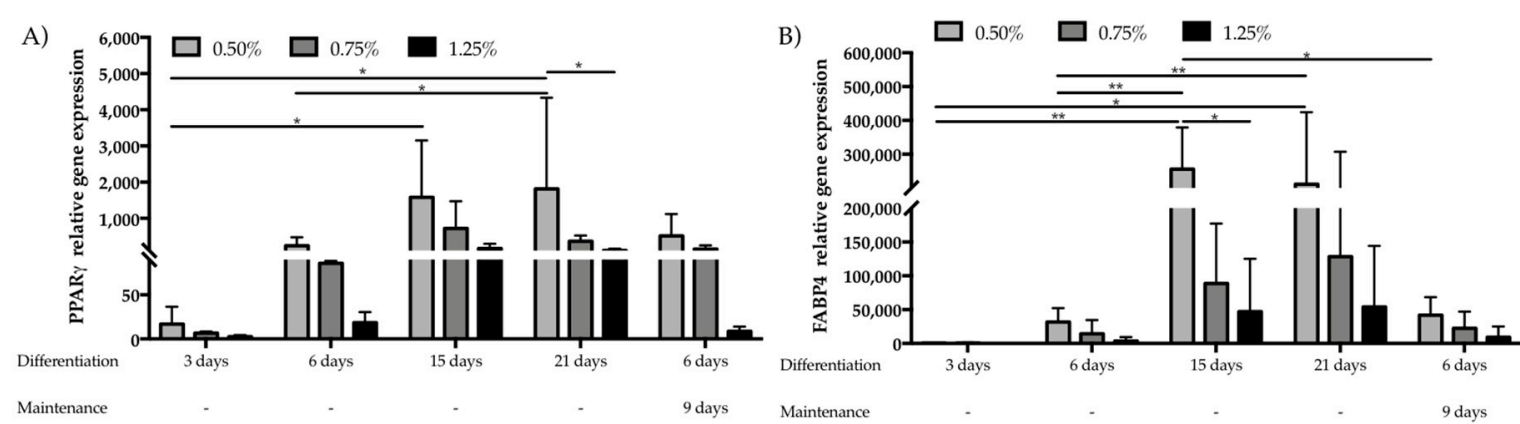

Figure 3. Relative expression of (A) PPAR $\gamma$ and (B) FABP4 genes in human adipose-derived stem cells within gellan gum hydrogel particles with different amounts of polymer $(0.5 \%, 0.75 \%$ and $1.25 \%)$ cultured in adipogenic induction medium for 3, 6, 15 and 21 days and for six days plus maintenance medium for 9 days. ${ }^{*} p<0.05$ and ${ }^{* *} p<0.01$.

To confirm the gene expression results and the hASCs capacity to differentiate towards adipogenic lineage within the GG hydrogel particles, the expression of PPAR $\gamma$ (Figure 4A) and FABP4 (Figure 4B) was evaluated at the protein level by immunocytochemistry. PPAR $\gamma$ expression started at day three and seemed to increase along the differentiation time (Figure 4A). The expression FABP4 was not detected until day 15 of culture in induction medium or after 6 days of culture in induction plus nine days in maintenance media. In comparison to day 15 of induction, it seems that a higher number of cells is expressing FABP4 at day 21 (Figure 4B). This trend was confirmed by Western blot (Figure 4C), although the semi-quantitative analysis did not reveal significant differences.

To further confirm the adipogenic phenotype of the cells along the differentiation within the produced hydrogel particles, the accumulation of neutral intracellular lipids was analysed through Nile Red staining (Figure 5). These lipids started to be seen at day six of induction medium and accumulate at higher amounts at longer induction time-points. Moreover, when maintenance medium was added after the six days of culture in induction medium, the levels of neutral lipids seem to be similar to those observed after 15 days of culture in induction medium. 


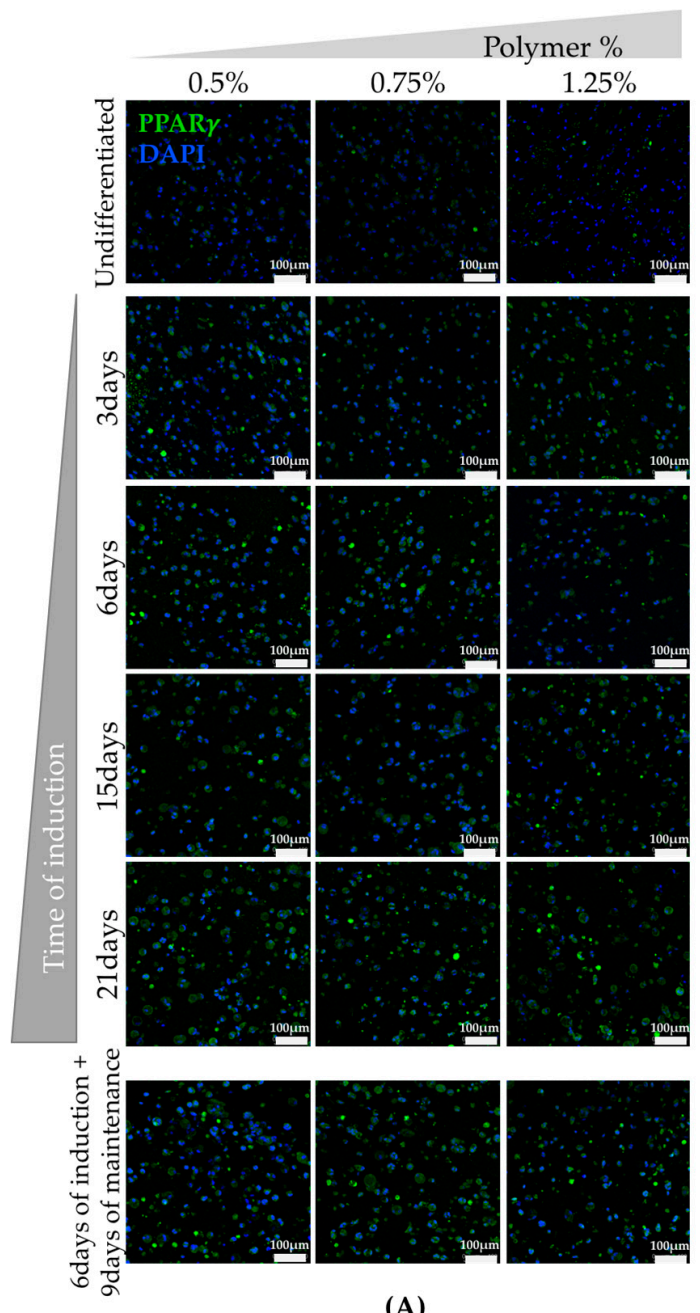

(A)

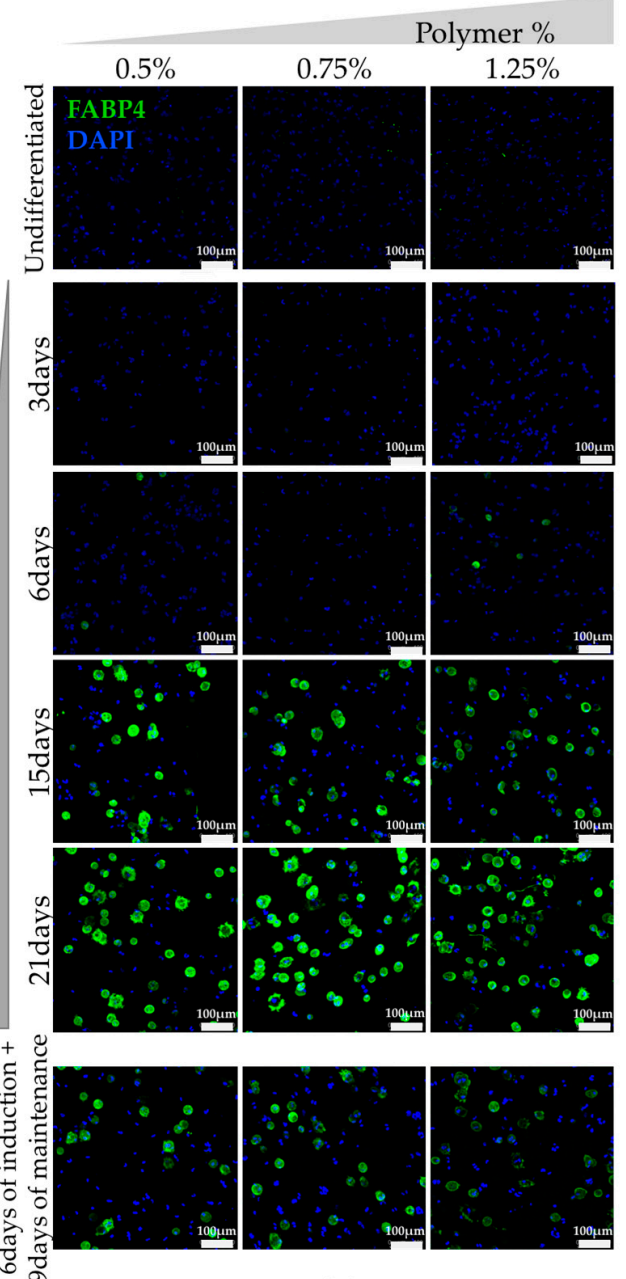

(B)

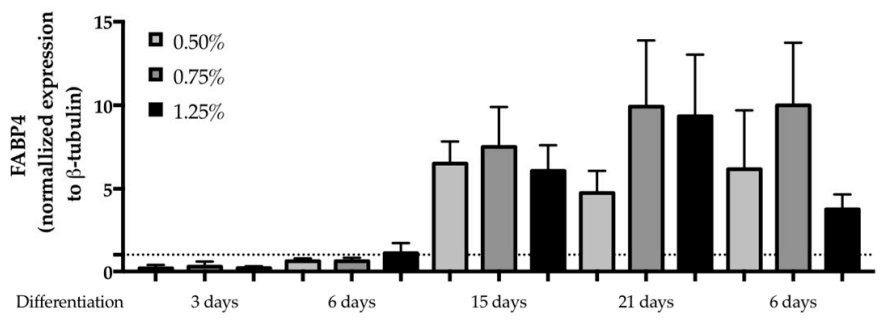

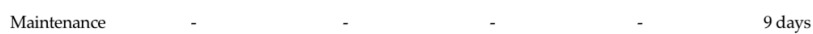

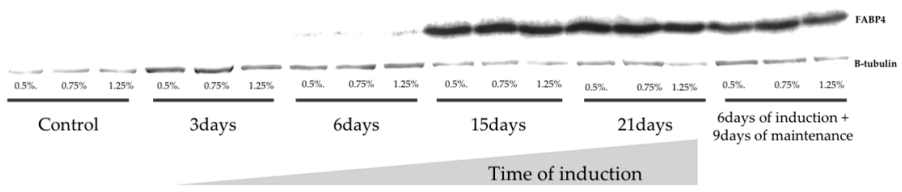

(C)

Figure 4. Adipogenic differentiation of human adipose-derived stem cells within gellan gum hydrogel particles with different amounts of polymer along the culture in adipogenic induction medium and after being cultured for six days in induction plus nine days in maintenance media. Representative confocal images of (A) PPAR $\gamma$ and (B) FABP4 expression (green). Cells nuclei were labelled with DAPI (blue). (C) Representative western Blot analysis of the expression of FABP4. Plotted data was normalized against beta-tubulin expression that was used as loading control. Scale bar $100 \mu \mathrm{m}$. 


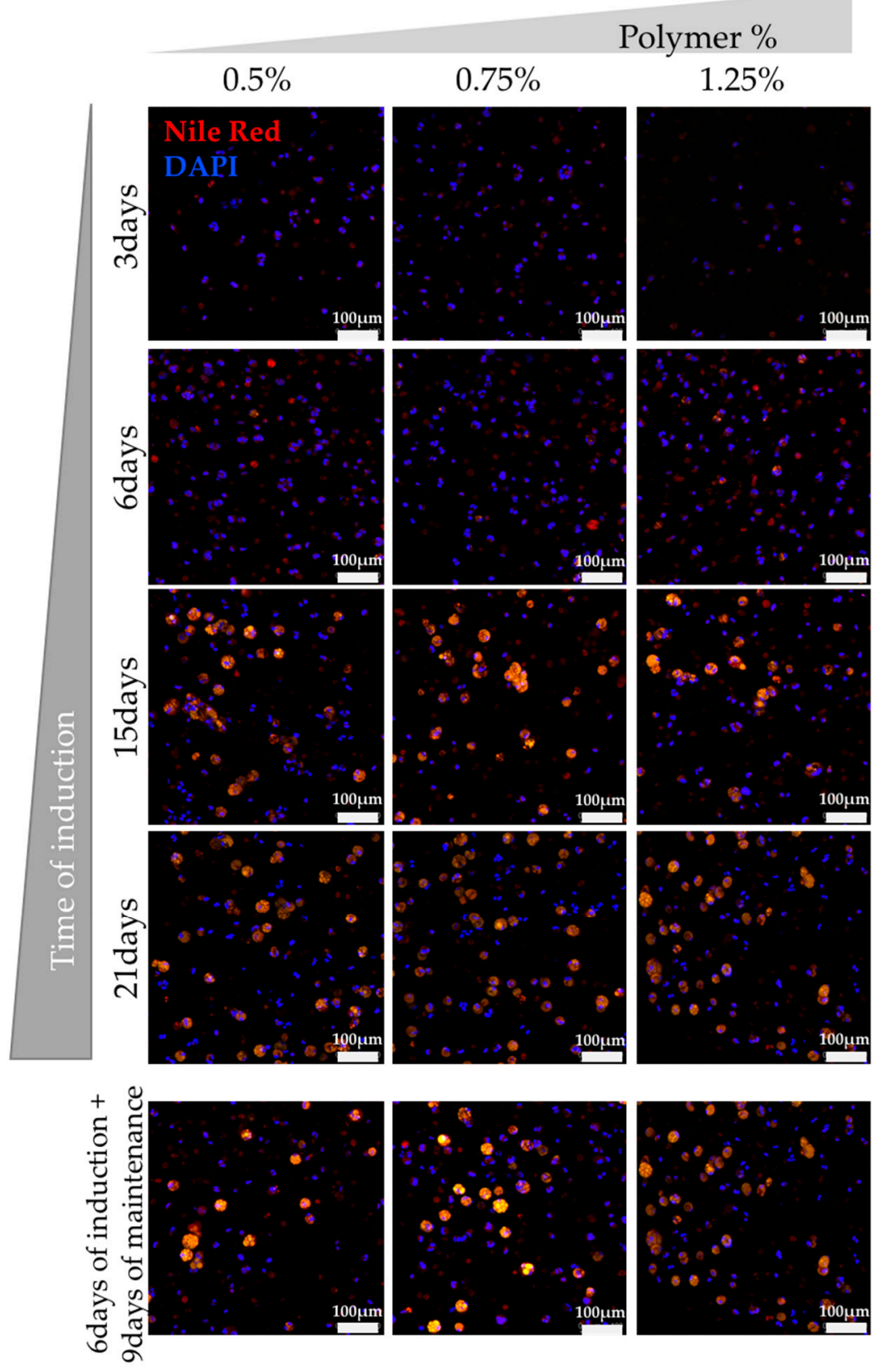

Figure 5. Neutral intracellular lipids accumulation in human adipose-derived stem cells-derived adipocytes within gellan gum hydrogel particles with different amounts of polymer along the culture in adipogenic induction medium and after being cultured for six days in induction plus nine days in maintenance media. Representative confocal images of cells stained with nile red (yellow). Nuclei were labelled with DAPI (blue). Polar cell membrane lipids are stained in red. Scale bar $100 \mu \mathrm{m}$.

\subsection{Assessment of TNF Alpha and ROCK Inhibitor Effect on Adipogenic Differentiation}

In order to prove the responsive behaviour of the proposed systems, the induction medium was supplemented with TNF $\alpha$ or ROCK inhibitor during the adipogenic differentiation (Figure 6A). Nile Red staining confirmed that the differentiation process is highly compromised in the presence of TNF alpha. On the other hand, in the presence of ROCK inhibitor the adipogenic differentiation occurs as previously observed (Figure 5). Moreover, the obtained results, both in the presence TNF alpha and ROCK inhibitor in the GG hydrogel particles, compare to those obtained in standard 2D drug screening culture conditions used as control (Figure 6B). 


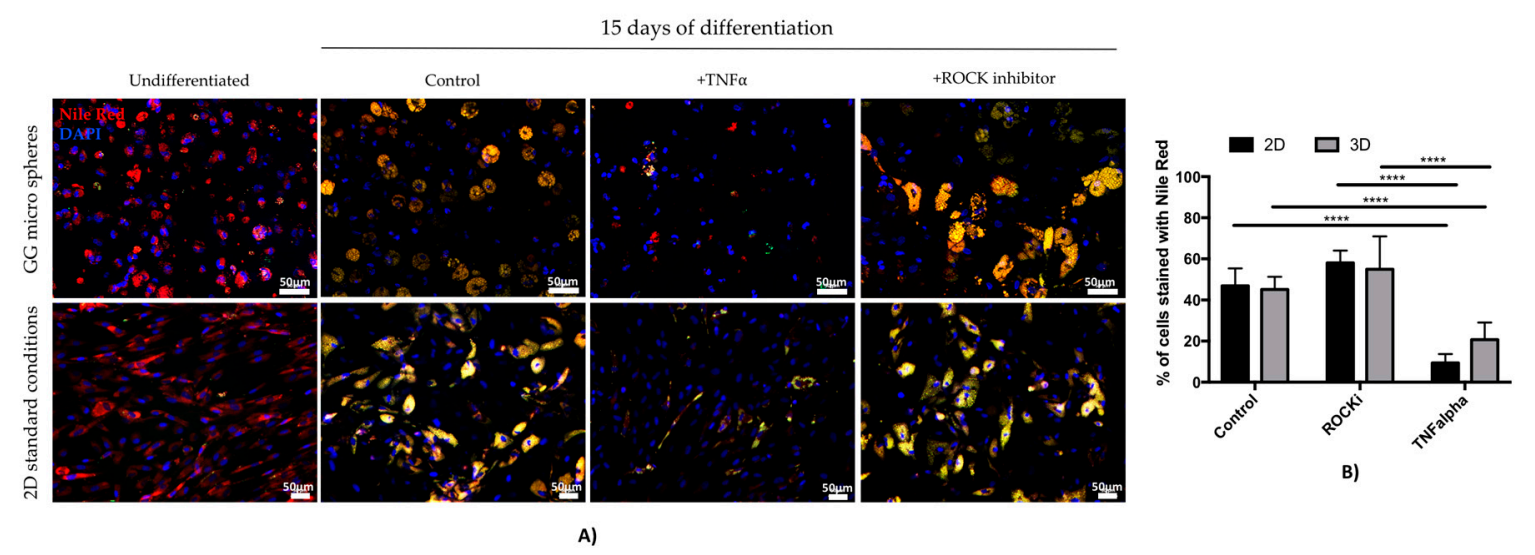

Figure 6. Assessment of the influence of TNF $\alpha(0.5 \mu \mathrm{g} / \mathrm{mL})$ and ROCK inhibitor $(50 \mu \mathrm{M})$ over the adipogenic differentiation of human adipose-derived stem cells within $0.5 \%$ gellan gum hydrogel particles after 15 days of culture in induction medium. (A) Representative images of nile red staining (polar cell membrane lipids are stained in red), and respective (B) quantification of the percentage of stained cells in relation to the total number of cells. Scale bar $50 \mu \mathrm{m} .{ }^{* * *} p<0.0001$.

\section{Discussion and Conclusions}

The development of adipose tissue equivalents has been relying on different strategies that, despite the promising results, also present some limitations, namely in terms of achieved degree of differentiation and stability of the constructs. In this work, we intended to tackle these limitations by exploring the natural features of GG to recreate stable and reliable adipose-like microtissues. Therefore, we tuned GG hydrogels properties by changing the polymer content generating constructs with Young's moduli between 2.7 and $12.9 \mathrm{kPa}$. Formulations with $0.5 \%$ and $0.75 \%$ of GG showed a modulus in the range of the native adipose tissue that varies between 3 and $7 \mathrm{kPa}$ [25-28]. In opposition, hydrogel formulations with $1.25 \%$ of GG have a modulus of approximately $12 \mathrm{kPa}$, in the highest limit reported in the literature for subcutaneous adipose tissue [28]. The amount of polymer also affected the mass loss profile of the GG particles in an inverse proportion as expected by our previous results [21], although the total amount of mass loss occurs within the first day. This is likely to correlate mostly to the non-polymeric content of GG hydrogel, rather than to the hydrolysis of the polymer. In fact, when the hydrogels are placed in the saline solution an exchange of ions/water from the culture medium, in which the hydrogels were prepared, to the saline solution occurs resulting in mass loss within the first day. This explanation is also consistent with the fact that when the polymeric/non-polymeric ratio is lower ( $0.5 \%$ GG hydrogel particles), the percentage of lost mass is higher due to the higher amount of water coming out from the hydrogels to reach an equilibrium state. Moreover, the absence of hydrolysis and consequently the lack of degradation of the polymer can be confirmed by the dried mass of the hydrogel particles at the end time point, in which the particles with successively lower polymeric content seem to gain weight. This is due to the amount of salts, in addition to the polymeric content that keeps constant, that is kept in the dried structure and that is higher for the formulation with lower polymeric content in which a higher water loss was observed.

It is also been shown that the mechanical properties of 3D microenvironments influence adipogenic differentiation although differently depending on the use of adipose tissue decellularized [29] or biomaterials-based [7] matrices. We confirmed the adipogenic differentiation of hASCs within the GG hydrogels by assessing the expression profile of early (PPAR $\gamma$ ) and late (FABP4) adipogenic markers in the different formulations and along the time. Although the action mechanism of PPAR $\gamma$ is not fully understood, PPAR $\gamma$ is a transcriptional factor that is highly expressed in adipocytes and is a major regulator of adipogenesis through the modulation of other adipogenic-related genes expression, such as FABP4 [30]. Having this in consideration, the upregulation of the expression of this gene as the induction period increases confirmed the triggering of the adipogenic differentiation and its 
maintenance in induction medium. While we could expect a downregulation after some time of culture, the presence of PPAR $\gamma$ agonist troglitazone in the induction medium might in part be responsible for the observed upregulated expression of PPAR $\gamma$ [31] at latter times. This is also supported by the fact that the level of upregulation, in the condition using induction and maintenance (without troglitazone) media, was lower, particularly for the $0.5 \%$ GG hydrogel particles, and comparable to the same time period only in induction medium. The gene expression results showed that FABP4 expression is favoured in hydrogels with lower polymeric content and hence in more compliant hydrogels, matching native tissue values [25-28], which corroborates with the literature [7,29]. Despite the trend for FABP4 mRNA expression results, the semi-quantitative analysis of the amount of FABP4 protein did not reveal significant differences, which might be associated to protein regulation at the posttranscriptional level [32].

Since the success of soft tissue reconstruction approaches is currently limited by the $40-60 \%$ volume retention of autologous adipose tissue, depending on its source [33,34], long-term stability of adipose-like tissues is crucial [35]. Proposed biomaterial-based approaches have been exploring several natural polymers such as collagen and alginate, however their degradability might be too fast. In opposition, GG degradation by hydrolysis, which is associated to long-term stability, represents an advantage over these materials for adipose tissue reconstruction. As mentioned before, adipose-like microtissues can be also of great relevance as in vitro models for drug screening or for unravelling the mechanistic of adipose tissue-associated pathologies. Considering this, we were able to demonstrate that the adipose-like microtissues that we generated are responsive to TNF $\alpha$ and Y-27632 (ROCK inhibitor). Y-27632 is a well-known ROCK kinase inhibitors reported as an enhancer of adipogenesis [36], while TNF $\alpha$ is known as a negative regulator of adipogenesis by preventing the early induction of PPAR $\gamma$ and C/EBP $\alpha$ expression [37,38]. Therefore we were able to confirm that our $3 \mathrm{D}$ constructs respond as expected, as shown by the absence presence of neutral intracellular lipids accumulation, respectively in the presence of ROCK inhibitor and TNF $\alpha$. Moreover, the results of our 3D system compare with those obtained in the standard 2D culture conditions confirming the potential of the developed microtissues to be used as in vitro drug screening platforms.

In conclusion, independently of the mechanical properties of the hydrogels, we developed stable and injectable GG-based adipose-like microtissues that can be considered for soft tissue reconstruction, as well as $3 \mathrm{D}$ in vitro tissue analogues for drug screening.

Supplementary Materials: The following are available online at http:/ / www.mdpi.com/2306-5354/5/3/52/s1, Figure S1: Capacity of human adipose-derived stem cells (hASCs) to differentiate in different lineages; Table S1: Phenotypic characterization of human adipose-derived stem cells prior differentiation through flow cytometry.

Author Contributions: Formal analysis, M.E.L.L., L.P.d.S., C.H. and A.F.C.; Funding acquisition, R.L.R. and A.P.M.; Methodology, M.E.L.L., L.P.d.S., C.H., A.F.C. and A.P.M.; Project administration, R.L.R. and A.P.M.; Supervision, A.P.M.; Writing—original draft, M.E.L.L. and L.P.d.S.; Writing—review \& editing, R.L.R. and A.P.M.

Funding: This research was funded by Fundação para a Ciência e a Tecnologia: IF/00945/2014.

Acknowledgments: The authors would like to acknowledge Daniel Rodrigues, Ângela Ferreira and Joana Pires for the support on experimental assays and to FSE/POCH-PD/169/2013, NORTE-08-5369-FSE-000037 (MELL), POCI-01-0145-FEDER-007038-UMINHO/BPD/44/2016 (LdS); Portuguese Foundation for Science and Technology (FCT) for grant SFRH/BPD/109595/2015 (AFC); IF/00945/2014 (APM); The Discoveries Centre for Regenerative and Precision Medicine (H2020-WIDESPREAD-2014-1-739572).

Conflicts of Interest: The authors declare no conflict of interest.

\section{References}

1. Pope, B.D.; Warren, C.R.; Parker, K.K.; Cowan, C.A. Microenvironmental control of adipocyte fate and function. Trends Cell Biol. 2016, 26, 745-755. [CrossRef] [PubMed]

2. Ouchi, N.; Parker, J.L.; Lugus, J.J.; Walsh, K. Adipokines in inflammation and metabolic disease. Nat. Rev. Immunol. 2011, 11, 85-97. [CrossRef] [PubMed]

3. Patrick, C.W. Tissue engineering strategies for adipose tissue repair. Anat. Rec. 2001, 263, 361-366. [CrossRef] [PubMed] 
4. $\quad$ Chen, Y.S.; Chen, Y.Y.; Hsueh, Y.S.; Tai, H.C.; Lin, F.H. Modifying alginate with early embryonic extracellular matrix, laminin and hyaluronic acid for adipose tissue engineering. J. Biomed. Mater. Res. Part A 2015. [CrossRef] [PubMed]

5. Hsueh, Y.S.; Chen, Y.S.; Tai, H.C.; Mestak, O.; Chao, S.C.; Chen, Y.Y.; Shih, Y.; Lin, J.F.; Shieh, M.J.; Lin, F.H. Laminin-alginate beads as preadipocyte carriers to enhance adipogenesis in vitro and in vivo. Tissue Eng. Part A 2016. [CrossRef]

6. Rossi, E.; Gerges, I.; Tocchio, A.; Tamplenizza, M.; Aprile, P.; Recordati, C.; Martello, F.; Martin, I.; Milani, P.; Lenardi, C. Biologically and mechanically driven design of an RGD-mimetic macroporous foam for adipose tissue engineering applications. Biomaterials 2016, 104, 65-77. [CrossRef] [PubMed]

7. Davidenko, N.; Campbell, J.J.; Thian, E.S.; Watson, C.J.; Cameron, R.E. Collagen-hyaluronic acid scaffolds for adipose tissue engineering. Acta Biomater. 2010, 6, 3957-3968. [CrossRef] [PubMed]

8. Yao, R.; Zhang, R.; Lin, F.; Luan, J. Injectable cell/hydrogel microspheres induce the formation of fat lobule-like microtissues and vascularized adipose tissue regeneration. Biofabrication 2012, 4, 045003. [CrossRef] [PubMed]

9. Girandon, L.; Kregar-Velikonja, N.; Bozikov, K.; Barlic, A. In vitro models for adipose tissue engineering with adipose-derived stem cells using different scaffolds of natural origin. Folia Biol. (Praha) 2011, 57, 47-56. [PubMed]

10. Korurer, E.; Kenar, H.; Doger, E.; Karaoz, E. Production of a composite hyaluronic acid/gelatin blood plasma gel for hydrogel-based adipose tissue engineering applications. J. Biomed. Mater. Res. Part A 2014, 102, 2220-2229. [CrossRef] [PubMed]

11. Bellas, E.; Marra, K.G.; Kaplan, D.L. Sustainable three-dimensional tissue model of human adipose tissue. Tissue Eng. Part C Methods 2013, 19, 745-754. [CrossRef] [PubMed]

12. Clevenger, T.N.; Hinman, C.R.; Ashley Rubin, R.K.; Smither, K.; Burke, D.J.; Hawker, C.J.; Messina, D.; Van Epps, D.; Clegg, D.O. Vitronectin-based, biomimetic encapsulating hydrogel scaffolds support adipogenesis of adipose stem cells. Tissue Eng. Part A 2016, 22, 597-609. [CrossRef] [PubMed]

13. Flynn, L.E. The use of decellularized adipose tissue to provide an inductive microenvironment for the adipogenic differentiation of human adipose-derived stem cells. Biomaterials 2010, 31, 4715-4724. [CrossRef] [PubMed]

14. Abbott, R.D.; Wang, R.Y.; Reagan, M.R.; Chen, Y.; Borowsky, F.E.; Zieba, A.; Marra, K.G.; Rubin, J.P.; Ghobrial, I.M.; Kaplan, D.L. The use of silk as a scaffold for mature, sustainable unilocular adipose 3D tissue engineered systems. Adv. Healthc. Mater. 2016, 5, 1667-1677. [CrossRef] [PubMed]

15. Stevens, L.R.; Gilmore, K.J.; Wallace, G.G.; in het Panhuis, M. Tissue engineering with gellan gum. Biomater. Sci. 2016, 4, 1276-1290. [CrossRef] [PubMed]

16. Matricardi, P.; Cencetti, C.; Ria, R.; Alhaique, F.; Coviello, T. Preparation and characterization of novel Gellan gum hydrogels suitable for modified drug release. Molecules 2009, 14, 3376-3391. [CrossRef] [PubMed]

17. Oliveira, J.T.; Gardel, L.S.; Rada, T.; Martins, L.; Gomes, M.E.; Reis, R.L. Injectable gellan gum hydrogels with autologous cells for the treatment of rabbit articular cartilage defects. J. Orthop. Res. 2010, 28, 1193-1199. [CrossRef] [PubMed]

18. Pereira, D.R.; Silva-Correia, J.; Caridade, S.G.; Oliveira, J.T.; Sousa, R.A.; Salgado, A.J.; Oliveira, J.M.; Mano, J.F.; Sousa, N.; Reis, R.L. Development of gellan gum-based microparticles/hydrogel matrices for application in the intervertebral disc regeneration. Tissue Eng. Part C Methods 2011, 17, 961-972. [CrossRef] [PubMed]

19. Silva, N.A.; Cooke, M.J.; Tam, R.Y.; Sousa, N.; Salgado, A.J.; Reis, R.L.; Shoichet, M.S. The effects of peptide modified gellan gum and olfactory ensheathing glia cells on neural stem/progenitor cell fate. Biomaterials 2012, 33, 6345-6354. [CrossRef] [PubMed]

20. Wang, C.; Gong, Y.; Lin, Y.; Shen, J.; Wang, D.A. A novel gellan gel-based microcarrier for anchoragedependent cell delivery. Acta Biomater. 2008, 4, 1226-1234. [CrossRef] [PubMed]

21. da Silva, L.P.; Cerqueira, M.T.; Sousa, R.A.; Reis, R.L.; Correlo, V.M.; Marques, A.P. Engineering cell-adhesive gellan gum spongy-like hydrogels for regenerative medicine purposes. Acta Biomater. 2014, 10, 4787-4797. [CrossRef] [PubMed]

22. Pereira Da Silva, L.; Teixeira Cerqueira, M.; Romero Amandi De Sousa, R.P.; Pinto Marques, A.M.; Correlo Da Silva, V.M.; Goncalves Dos Reis, R.L. Gellan Gum Spongy-Like Hydrogel, its Preparation and Biomedical Applications Thereof. U.S. Patent 14782812, 9 April 2014. 
23. Cerqueira, M.T.; Pirraco, R.P.; Santos, T.C.; Rodrigues, D.B.; Frias, A.M.; Martins, A.R.; Reis, R.L.; Marques, A.P. Human adipose stem cells cell sheet constructs impact epidermal morphogenesis in full-thickness excisional wounds. Biomacromolecules 2013, 14, 3997-4008. [CrossRef] [PubMed]

24. Yu, G.; Floyd, Z.E.; Wu, X.; Hebert, T.; Halvorsen, Y.D.C.; Buehrer, B.M.; Gimble, J.M. Adipogenic differentiation of adipose-derived stem cells. In Adipose-Derived Stem Cells: Methods and Protocols; Springer: Berlin, Germany, 2011; pp. 193-200.

25. Samani, A.; Bishop, J.; Luginbuhl, C.; Plewes, D.B. Measuring the elastic modulus of ex vivo small tissue samples. Phys. Med. Biol. 2003, 48, 2183-2198. [CrossRef] [PubMed]

26. Comley, K.; Fleck, N.A. A micromechanical model for the Young's modulus of adipose tissue. Int. J. Solids Struct. 2010, 47, 2982-2990. [CrossRef]

27. Samani, A.; Zubovits, J.; Plewes, D. Elastic moduli of normal and pathological human breast tissues: An inversion-technique-based investigation of 169 samples. Phys. Med. Biol. 2007, 52, 1565. [CrossRef] [PubMed]

28. Alkhouli, N.; Mansfield, J.; Green, E.; Bell, J.; Knight, B.; Liversedge, N.; Tham, J.C.; Welbourn, R.; Shore, A.C.; Kos, K.; et al. The mechanical properties of human adipose tissues and their relationships to the structure and composition of the extracellular matrix. Am. J. Physiol.-Endocrinol. Metab. 2013, 305, E1427-E1435. [CrossRef] [PubMed]

29. Yu, C.; Bianco, J.; Brown, C.; Fuetterer, L.; Watkins, J.F.; Samani, A.; Flynn, L.E. Porous decellularized adipose tissue foams for soft tissue regeneration. Biomaterials 2013, 34, 3290-3302. [CrossRef] [PubMed]

30. Mauney, J.R.; Nguyen, T.; Gillen, K.; Kirker-Head, C.; Gimble, J.M.; Kaplan, D.L. Engineering adipose-like tissue in vitro and in vivo utilizing human bone marrow and adipose-derived mesenchymal stem cells with silk fibroin 3D scaffolds. Biomaterials 2007, 28, 5280-5290. [CrossRef] [PubMed]

31. Rosen, E.D.; MacDougald, O.A. Adipocyte differentiation from the inside out. Nat. Rev. Mol. Cell Biol. 2006, 7, 885-896. [CrossRef] [PubMed]

32. Garin-Shkolnik, T.; Rudich, A.; Hotamisligil, G.S.; Rubinstein, M. FABP4 attenuates PPAR $\gamma$ and adipogenesis and is inversely correlated with PPAR $\gamma$ in adipose tissues. Diabetes 2014, 63, 900-911. [CrossRef] [PubMed]

33. Lorenz, H.P.; Hedrick, M.H.; Chang, J.; Mehrara, B.J.; Longaker, M.T. The impact of biomolecular medicine and tissue engineering on plastic surgery in the 21st century. Plast. Reconstr. Surg. 2000, 105, 2467-2481. [CrossRef] [PubMed]

34. Tanzi, M.C.; Farè, S. Adipose tissue engineering: State of the art, recent advances and innovative approaches. Expert Rev. Med. Devices 2009, 6, 533-551. [CrossRef] [PubMed]

35. Stosich, M.S.; Mao, J.J. Adipose tissue engineering from human adult stem cells: Clinical implications in plastic and reconstructive surgery. Plast. Reconstr. Surg. 2007, 119, 71-85. [CrossRef] [PubMed]

36. Noguchi, M.; Hosoda, K.; Fujikura, J.; Fujimoto, M.; Iwakura, H.; Tomita, T.; Ishii, T.; Arai, N.; Hirata, M.; Ebihara, K.; et al. Genetic and pharmacological inhibition of Rho-associated kinase II enhances adipogenesis. J. Biol. Chem. 2007, 282, 29574-29583. [CrossRef] [PubMed]

37. Cawthorn, W.P.; Heyd, F.; Hegyi, K.; Sethi, J.K. Tumour necrosis factor- $\alpha$ inhibits adipogenesis via a $\beta$-catenin/TCF4(TCF7L2)-dependent pathway. Cell Death Differ. 2007, 14, 1361-1373. [CrossRef] [PubMed]

38. Xu, H.; Sethi, J.K.; Hotamisligil, G.S. Transmembrane tumor necrosis factor (TNF)- $\alpha$ inhibits adipocyte differentiation by selectively activating TNF receptor 1. J. Biol. Chem. 1999, 274, 26287-26295. [CrossRef] [PubMed]

(C) 2018 by the authors. Licensee MDPI, Basel, Switzerland. This article is an open access article distributed under the terms and conditions of the Creative Commons Attribution (CC BY) license (http:/ / creativecommons.org/licenses/by/4.0/). 